\title{
Nonthermally activated exciton transport in crystalline organic semiconductor thin films
}

\author{
Anna K. Topczak, ${ }^{1, *}$ Tobias Roller, ${ }^{2}$ Bernd Engels, ${ }^{1}$ Wolfgang Brütting, ${ }^{3}$ and Jens Pflaum ${ }^{1,4, \dagger}$ \\ ${ }^{1}$ Julius-Maximillians University, Am Hubland, 97074 Würzburg, Germany \\ ${ }^{2}$ University of Stuttgart, Pfaffenwaldring 57, 70569 Stuttgart, Germany \\ ${ }^{3}$ University of Augsburg, Universitätsstraße 1, 86159 Augsburg, Germany \\ ${ }^{4}$ ZAE Bayern e.V., Am Hubland, 97074 Würzburg, Germany \\ (Received 23 January 2014; revised manuscript received 14 April 2014; published 16 May 2014)
}

\begin{abstract}
The temperature dependent exciton transport in the prototypical organic semiconductor di-indeno-perylene (DIP) is investigated by photoluminescence quenching. Analysis by an advanced diffusion model including interference and morphological aspects reveals an exciton diffusion length of about $60 \mathrm{~nm}$ at room temperature, which relates to the long-range order induced by the DIP molecular shape. Above $80 \mathrm{~K}$, singlet exciton transport is thermally activated with an energy of 10 to $20 \mathrm{meV}$. Below $80 \mathrm{~K}$, exciton motion becomes temperature independent and is supported by the crystalline structure of the transport layer in combination with the reduced phonon interaction.
\end{abstract}

DOI: 10.1103/PhysRevB.89.201203

PACS number(s): 68.35.bm, 68.55.am, 71.35.-y, 72.10.Di

With progressively improving power conversion efficiency, organic thin film devices have paved their way towards commercial optoelectronic applications [1]. In many of these, the exciton diffusion length is a crucial parameter for the overall performance. For instance, in bilayer organic photovoltaic (OPV) cells, the competition between the exciton diffusion length $L_{\mathrm{D}}$ and the absorption length $\alpha^{-1}$ is decisive for the applicable thickness range of the photoactive layers [2]. Disordered polymer films offer short exciton diffusion lengths of several nanometers since the much faster exciton relaxation on the conjugated segments outbalances the long distance exciton transfer via interchain hopping [2,3,4]. Therefore, thin films of small molecules with a higher degree of structural order are promising candidates to achieve enhanced exciton migration within their crystalline domains. Since the oscillator strength of dipole-allowed transitions reduces the lifetime of singlet excitons, diffusion lengths exceeding several tens of nanometers or even reaching the absorption length have yet to be reported.

As it became obvious from observations of a bandlike exciton motion in organic single crystals [5] that morphology significantly influences the diffusion characteristics of optical excitations, molecular model systems supporting coherent exciton transport in thin films are highly desired [6,7]. To aim for this challenge we have performed temperature dependent exciton transport studies on thin films of the prototypical organic semiconductor di-indeno-perylene (DIP), pictured in Fig. 2(b). Bilayer thin film cells of DIP, in combination with the electron acceptor $\mathrm{C}_{60}$, have shown superior performance, including fill factors above $70 \%$ and power conversion efficiencies of up to $3 \%$ [8]. Motivated by these results, we present a general approach on the determination of exciton transport properties by thickness dependent photoluminescence (PL) quenching studies, considering morphological aspects such as the grain size distribution, quencher penetration, and optical interference effects. As will be shown, this method reveals singlet exciton motion in crystalline DIP layers to

\footnotetext{
*atopczak@physik.uni-wuerzburg.de

†jpflaum@physik.uni-wuerzburg.de
}

be nonactivated at low temperatures, and thus offers insight into the material inherent transport mechanism. In comparison to other techniques facilitating the estimation of the exciton diffusion length, such as complementary analyses of the spectrally resolved photocurrent and absorption [7,9], PL measurements feature the advantage of investigating the intrinsic exciton transport properties without external influences by metal penetration, field-induced exciton dissociation, or recombination of charge carriers as, e.g., in OPV devices under operation [4,10-12]. Moreover, theoretical modeling of our experimental data benefits from the lack of approximations on the exciton diffusion and absorption lengths, which would cause constraints in the present case where $L_{\mathrm{D}}$ approaches $\alpha^{-1}$.

DIP thin films are grown by molecular beam deposition under high vacuum (base pressure $<10^{-7} \mathrm{mbar}$ ) on cleaned glass substrates at a growth rate of $2 \mathrm{~nm} / \mathrm{min}$. The resulting film structure is analyzed in detail by $x$-ray diffraction at various geometries. Bragg-Brentano scans confirm the previously reported (001) DIP lattice spacing of about $1.66 \mathrm{~nm}$ corresponding to an almost upright orientation of the molecules [13], and the long-range coherence of the lattice planes is indicated by pronounced Laue oscillations at the (001) Bragg peak [Fig. 1(a), left inset]. This orientation of the DIP molecules promotes vertical exciton transport due to a favored coupling of the molecular transition dipole moments $[14,15]$. Furthermore, the DIP film mosaicity is characterized by an averaged crystallite tilting of only $0.04^{\circ}$ along the surface normal, i.e., along the direction of exciton transport [right inset of Fig. 1(a)]. As a measure of the film quality we employ the ratio of specular versus diffuse intensity of the corresponding rocking scans [dark versus light shaded areas in the right inset of Fig. 1(a)], the latter being influenced by structural inhomogeneities within the organic layer [16]. As can be seen, this ratio peaks at a thickness of about $70 \mathrm{~nm}$, in agreement with the crystallite height determined by the Laue oscillations. Based on these data, a model of the underlying film structure has been deduced and is described by an error function representing the integrated Gaussian distribution of DIP crystallite heights [Fig. 1(b)].

For the PL studies step-wedged DIP samples covering a thickness range between 10 and $300 \mathrm{~nm}$ are half-side capped 


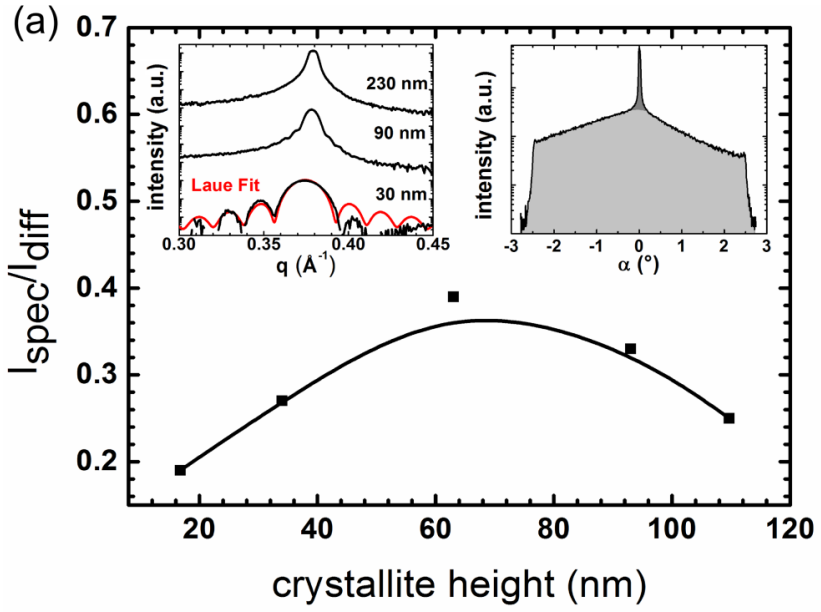

(b)

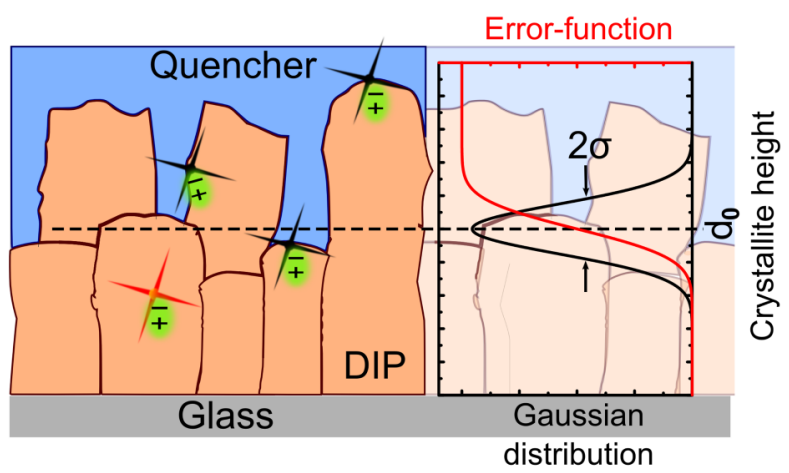

FIG. 1. (Color online) (a) The intensity ratio between specular (dark shaded area) and the diffuse scattering (light shaded area) of the respective rocking curves (upper right inset) has been considered as a measure of the structural quality of the DIP layers. Inset (left): Thickness dependent x-ray diffraction scans of the (001) DIP Bragg peak at $q_{z}=0.38 \AA^{-1}$, indicating the high crystallinity of the films along the direction of exciton transport. The red curve demonstrates a fit of the Laue oscillations. (b) Model of the DIP film texture, together with possible radiative (red cross) and nonradiative (black crosses) recombination processes. Integration of the Gaussian distribution of DIP crystallite heights (black curve) yields the error function (red curve).

by a vacuum sublimed $15 \mathrm{~nm}$ thick copper phthalocyanine $(\mathrm{CuPc})$ layer [Fig. 2(a)] acting as an exciton quencher due to its highest occupied molecular orbital (HOMO) and lowest unoccupied molecular orbital (LUMO) positions of 5.1 and $3.2 \mathrm{eV}$ with respect to those of DIP, 5.4 and $2.9 \mathrm{eV}[5,17]$. By choosing an interface to $\mathrm{CuPc}$ and therefore supporting exciton annihilation rather than dissociation, we minimize undesired effects by the interaction of excitons with free or trapped charges in the DIP transport layer [12]. Furthermore, CuPc absorption is negligible at the $532 \mathrm{~nm}$ excitation wavelength of the cw Nd:YAG laser used in this study, thereby avoiding the unintended effects on the exciton distribution in DIP. The PL intensity, $I_{\mathrm{PL}}$, of the DIP films, illuminated through the glass substrate, is measured by a sensitive CCD detector in combination with a spectrometer. At a given DIP layer thickness, the comparison of the relative PL intensity ratio $Q=$ $I_{\mathrm{PL}, Q} / I_{\mathrm{PL}, n Q}$ recorded on bare $\left(I_{\mathrm{PL}, n Q}\right)$ and CuPc-covered

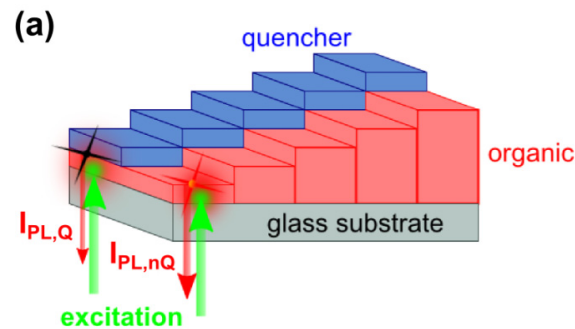

(b)

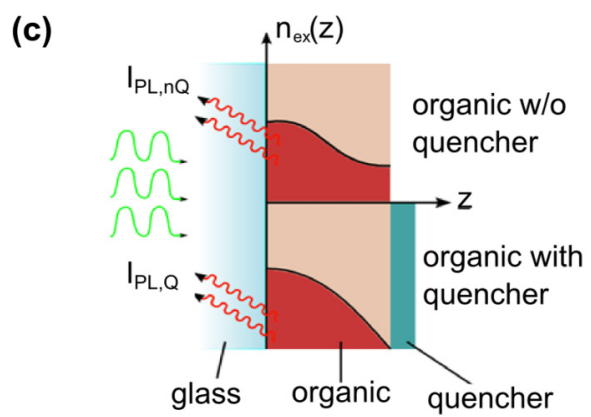

\section{DIP $\left(\mathrm{C}_{32} \mathrm{H}_{16}\right)$}

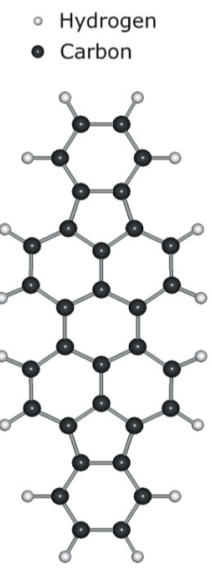

FIG. 2. (Color online) (a) Sample geometry: Step-wedged layer of DIP half-side covered with a $\mathrm{CuPc}$ quencher. The green arrows indicate the laser excitation whereas red arrows indicate the generated PL signals. (b) Structural model of the DIP molecule. (c) Schematic picture of the spatially dependent exciton generation $n_{\mathrm{ex}}(z)$ upon light absorption and the corresponding PL signals from the respective sample areas with and without quencher. The exciton generation profile $n_{\mathrm{ex}}(z)$ sketched by the red areas under the curves is related to the boundary conditions of no quenching (upper half) and of complete quenching at the back side (lower half).

$\left(I_{\mathrm{PL}, Q}\right)$ areas reveals contributions from nonradiative decay channels and from the quenching of excitons [2].

For films thinner than $L_{\mathrm{D}}$ the majority of excitons will reach the quenching interface within their radiative lifetime, thus strongly reducing the PL signal [Fig. 2(a)]. In contrast, for films much thicker than $L_{\mathrm{D}}$, the PL signal is expected to dependent only on the light absorption and therefore should be identical for free and quencher-covered areas. As a result, $L_{\mathrm{D}}$ of an organic semiconducting material can be determined by thickness dependent PL measurements. It is important to stress at this point that despite the spectral overlap between DIP emission and CuPc quencher absorption [18], contributions by fluorescence resonance energy transfer (FRET) to the transport and quenching of excitons are of minor importance for the layer thicknesses and $L_{\mathrm{D}}$ values under study [19], and are inherently included in the quenching quality factor introduced below. However, the situation becomes more complex for real samples, especially if the absorption layer thickness approaches the excitation wavelength $\lambda$ within the organic material. For thicknesses equal to or larger than $\lambda / 4 n$, interference effects have to be taken into account [20,21], requiring detailed information on the anisotropic refraction index $n$ of the organic material. Therefore, to quantify exciton quenching one has to calculate at first the intensity profile of incident light, including interference effects across the sample, yielding the initial exciton density. With $n(z, t)$ being the local exciton density at time $t$, the one-dimensional (1D) continuity 
equation reads

$$
\frac{\partial n(z, t)}{\partial t}=D \frac{\partial^{2} n(z, t)}{\partial z^{2}}-\frac{n(z, t)}{\tau}+G(z, t) .
$$

At a given exciton lifetime $\tau$, the exciton generation term $G(z, t)$ under constant illumination, i.e., at steady state conditions $\frac{\partial n(z, t)}{\partial t}=0$, is proportional to the intensity profile of the interfering light. Appropriate boundary conditions can be rationalized by either neglecting quenching, $\left.\frac{\partial n(z, t)}{\partial z}\right|_{z=0}=0$, or by assuming complete quenching, $n(z, t)=0$, at the respective interfaces. These situations are schematically depicted in Fig. 2(c). Real samples will always operate between these two extremes, i.e., partial quenching might occur at the organic/air interface, for instance, by surface states, as well as imperfect quenching at the organic/quencher interface. For perfect quenching the absolute value of the slope and, accordingly, the exciton concentration gradient will reach its maximum $S_{\max }$ at the quencher interface. This provides a measure for the quenching quality $V=S / S_{\max }$, where $S$ is the slope at the organic/quencher interface in the case of incomplete quenching. Obviously, $V$ takes values between 0 and 1 . As for more complex intensity profiles, Eq. (1) can no longer be solved analytically, we employed an ansatz based on the superposition of Dirac delta functions $G(z)=\delta\left(z-z_{0}\right) / \tau$ as generation term yielding the PL signal for any given $I\left(z_{0}\right)$ :

$$
I_{\mathrm{PL}}=k \int_{0}^{d} I\left(z_{0}\right)\left[1-V \frac{\cosh \left(z_{0} / L_{D}\right)}{\cosh \left(d / L_{D}\right)}\right] d z_{0} .
$$

Here we used the common definition of the exciton diffusion length, $L_{\mathrm{D}}=\sqrt{D \tau}$.

The thickness dependent quenching ratio $Q$ measured at room temperature (RT) is displayed in Fig. 3. Exemplarily, the spectrally resolved PL signal of a $120 \mathrm{~nm}$ thick DIP layer is included in the inset and highlights the strong decay of $I_{\mathrm{PL}, Q}$ vs $I_{\mathrm{PL}, n Q}$. Three distinct features can be identified: First, $Q$ does not approach zero even for $d_{\text {DIP }} \rightarrow 0$, suggesting imperfect quenching at the $\mathrm{DIP} / \mathrm{CuPc}$ interface. Second, the oscillatory thickness behavior of $Q$ refers to the presence of interference effects. Third, even for film thicknesses of $300 \mathrm{~nm}$, $Q$ is still below 0.6, indicating the existence of a significant, almost thickness independent, quenching contribution. To relate these findings to the underlying film morphology, complementary studies on the temperature dependent PL have been carried out on DIP layers of various thicknesses. The inset in Fig. 4 shows the photoluminescence intensity normalized to $I_{\mathrm{PL}, n Q}$ for a $30 \mathrm{~nm}$ thin DIP film with and without a quenching layer and exemplifies the strong decay of $I_{\mathrm{PL}, Q}$ with decreasing temperature. The overall temperature dependent relative quenching $Q$ normalized to its room temperature value is displayed in Fig. 4. Above $80 \mathrm{~K}$, the fluorescence arising from the free $\left(I_{\mathrm{PL}, n}\right)$ and the CuPc-covered $\left(I_{\mathrm{PL}, Q}\right)$ DIP volume is thermally activated. The energy related to the $I_{\mathrm{PL}, n Q}$ signal as determined by an Arrhenius fit shows a distinct increase with thickness from 12 to about $21 \mathrm{meV}$, and thus indicates that a steeper concentration gradient, occurring for thinner films, reduces the effective energy required for exciton migration [22]. In addition, this is confirmed by the quencher-capped $30 \mathrm{~nm}$ thick DIP film whose PL activation energy is about four times smaller than that of the uncovered

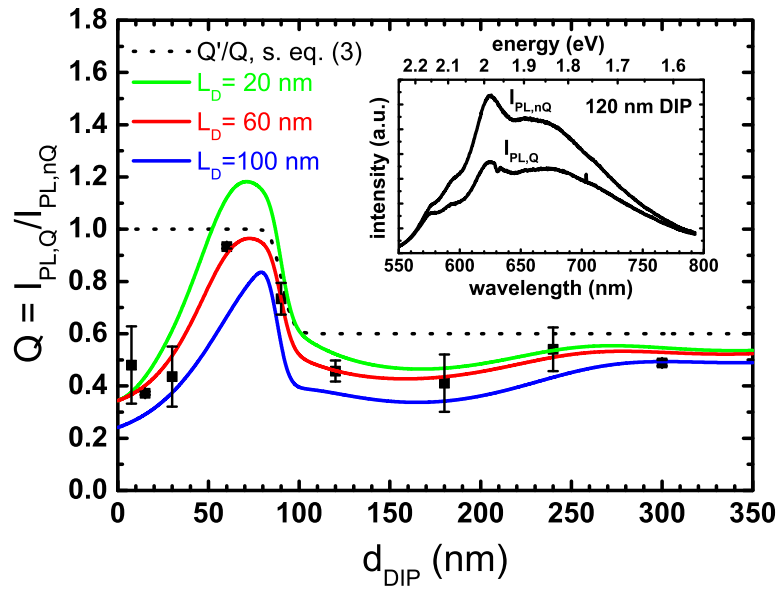

FIG. 3. (Color online) The fit (red line) corresponds to the advanced diffusion model discussed in the text. Accounting for interference effects and penetration at the DIP/quencher interface this model yields an exciton diffusion length $L_{\mathrm{D}}$ of $60 \mathrm{~nm}$ (goodness of fit $\chi^{2}$ : 0.051 ). The green and blue curves represent simulations for smaller $\left(L_{\mathrm{D}}=20 \mathrm{~nm}\right.$; goodness of fit $\left.\chi^{2}: 0.194\right)$ and larger diffusion lengths $\left(L_{\mathrm{D}}=100 \mathrm{~nm}\right.$; goodness of fit $\left.\chi^{2}: 0.293\right)$ reported in literature $[7,15]$. The larger the $L_{\mathrm{D}}$, the smaller the maximum of the relative quenching at $80 \mathrm{~nm}$. The inset displays the spectrally resolved PL signal of a $120 \mathrm{~nm}$ thick DIP layer with and without $\mathrm{CuPc}$ quencher, demonstrating the pronounced decline of $I_{\mathrm{PL}, Q}$ caused by exciton quenching.

film. For DIP films thicker than $100 \mathrm{~nm}$ the activation energy of $21 \mathrm{meV}$ is the same for bare and quencher-covered areas and, therefore, refers to exciton transport within the DIP crystallites.

In analogy to charge carrier transport studies on long-range ordered molecular stacks [23], we attribute the temperature dependent fluorescence behavior to inelastic exciton-phonon

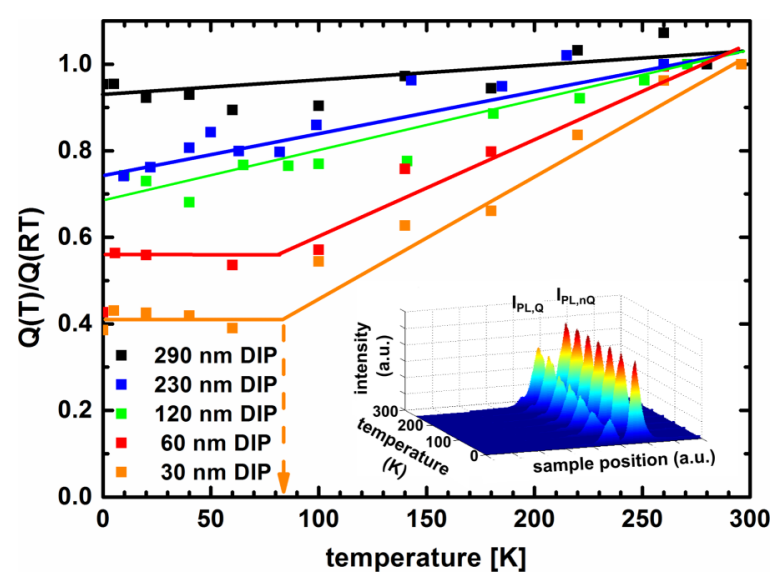

FIG. 4. (Color online) Relative quenching $Q$ as a function of temperature for five representative DIP layer thicknesses normalized to the $Q$ value at room temperature. Thin DIP layers show the signature of a thermally activated relative quenching above $80 \mathrm{~K}$, whereas below this temperature, the quenching ratio remains constant. Inset: Evolution of the temperature dependent PL intensity of a $30 \mathrm{~nm}$ thick DIP layer normalized to $I_{\mathrm{PL}, n Q}$, indicating the distinct quenching with decreasing temperature. 
scattering [24] constituting a nonradiative decay channel for the excited states. This assumption is corroborated by the observed activation energies which coincide with low-energy phonon modes in polyaromatic single crystals [23,25]. Furthermore, as it becomes obvious from Fig. 4, the $Q(T) / Q(\mathrm{RT})$ behavior is characterized by a pronounced decrease with temperature for thin DIP films, followed by a nonactivated transport regime below $80 \mathrm{~K}$. We suggest the latter to be caused by freezing out the nonlocal phonon modes to their ground state, i.e., causing the inelastic exciton-phonon scattering to become almost temperature independent and thus supporting the motion of excitonic species within the crystalline DIP domains [26]. In contrast, with increasing thickness, $Q$ approximates an almost constant behavior over the entire temperature range. This observation points directly at the morphological influence on the exciton transfer which can be understood by the previously discussed spatial extension of DIP crystallites along the transport direction, i.e., normal to the (001) plane [Fig. 1(b)]. For thick films $\left(d_{\text {DIP }}>100 \mathrm{~nm}\right)$ nonradiative as well as radiative recombination processes mainly take place within the single crystalline domains, due to energy barriers imposed at the grain boundaries. However, for thin films $\left(d_{\text {DIP }}<100 \mathrm{~nm}\right)$ with crystallites extending to the entire film thickness, exciton transport and recombination are substantially determined by the $\mathrm{CuPc}$ quenching interface. Consequently, the variation with temperature is mainly governed by the $I_{\mathrm{PL}, Q}$ contribution to $Q$. This model is supported by the fact that for thin DIP films the temperature independent $Q$ value below $80 \mathrm{~K}$ is significantly smaller than for thick layers as quenching is still present in the case of the former. For the latter, in contrast, excitons are no longer capable of reaching the $\mathrm{CuPc}$ quencher interface and relax to the ground state by the same mechanisms as for the uncovered DIP sections. In this case, the intrinsic crystallite size, which agrees very well with the optimized thickness deduced by x-ray diffraction from the $I_{\text {spec }} / I_{\text {diff }}$ ratio [Fig. 1(a)], correlates with a pronounced three-dimensional (3D) island growth (Volmer-Weber growth) accompanied by an enhanced surface roughening, as confirmed by detailed $\mathrm{x}$-ray structural analyses and atomic force microscopy (AFM) [27]. As indicated in Fig. 1(b), this topography promotes the penetration of the top-deposited quencher material at the grain boundaries, leading to an effective in-plane quenching of diffusing excitons and thereby to a significant contribution to the PL intensity reduction of about $50 \%$, even for thicknesses exceeding $250 \mathrm{~nm}$. It can be rationalized that this trend is supported by the good overlap of the electronic wave function and the large molecular transition dipole within the (001) plane [28], presumably entailing the lateral components of the diffusivity tensor to be of comparable size as the out-of-plane component [14]. A lower limit for the lateral diffusion length $L_{\mathrm{D}}$ of several tens of nanometers has been reported which overall matches our observation [29]. Summarizing these results, we suggest a relation to account for the morphological impact of real quencher interfaces on the excitonic processes, thereby justifying the aforementioned error function:

$$
Q^{\prime}=Q\left[\frac{1+R}{2}-\left(\frac{1-R}{2}\right) \operatorname{erf}\left(\frac{d-d_{0}}{\sigma(d)}\right)\right]
$$

The average crystallite height and surface roughness are described by $d_{0}$ and $\sigma(d)$, whereas $R$ empirically considers all effects that limit the relative quenching for thick layers. Modeling the thickness dependent $Q$ by our full exciton diffusion model, comprising effects of optical interference and quencher interface morphology, reveals the fit shown in Fig. 3 and an $L_{\mathrm{D}}$ of $60 \mathrm{~nm}$ for DIP at RT. Explicitly accounting for both effects, this value somewhat differs from previous data of about $100 \mathrm{~nm}$ obtained by spectral photocurrent measurements in combination with a diffusion model of Feng and Ghosh [7,9]. A deviation from the reported $L_{\mathrm{D}}$ values of $20 \mathrm{~nm}$ deduced from spectrally resolved PL quenching studies [15] indicates the impact of the underlying film morphology on the exciton transport, as it becomes obvious from differences in the XRD spectra of the corresponding thin film samples. To illustrate the effect of various diffusion lengths on the relative quenching and, thereby, to prove the sensitivity of our approach, Fig. 3 displays the $Q\left(d_{\text {DIP }}\right)$ behavior for these three different diffusion lengths. For small $L_{\mathrm{D}}$, the thickness dependent behavior of the relative quenching is governed by the light intensity distribution inside the layer. Values of $Q$ larger than 1 originate from the interference pattern at small $L_{\mathrm{D}}$. In the case of large $L_{\mathrm{D}}$, in particular, if the diffusion length exceeds the absorption depth $\alpha^{-1}$, interference effects become negligible and $Q$ is only influenced by the error function as well as the exponential absorption profile. The larger the $L_{\mathrm{D}}$, the less pronounced is the maximum of $Q$ at $80 \mathrm{~nm}$. Comparing $L_{\mathrm{D}}$ with the estimated average crystallite height of $d_{0}=88 \mathrm{~nm}$ renders the correlation between these two properties evident. Finally, the discussed transport model also comprises the thickness dependent interface roughness which, according to the rapid roughening observed for DIP thin films [27], scales as

$$
\sigma(d)=a d^{\beta} \text { with } \beta=0.70 \text { and } a=0.3 \text {. }
$$

In summary, we have investigated the exciton transport in long-range ordered molecular thin films by their PL quenching behavior, explicitly accounting for interference effects as well as for the structural properties at the respective quencher interface. Based on an advanced diffusion model, in combination with complementary morphological data, we were able to describe the thickness and temperature dependent exciton transport in DIP as being archetypical for the class of polyaromatic organic semiconductors. The estimated diffusion length of $60 \mathrm{~nm}$ at RT is in striking agreement with the average DIP crystallite height and, together with the observation of a nonactivated transport of singlet excitons at low temperatures, indicates the decisive role of structural order on the exciton transport. With the prospect of implementation in highefficiency organic photovoltaics, our results demonstrate the need for morphological control.

Financial support by the Deutsche Forschungsgemeinschaft (priority program SPP1355 and research unit FOR1809) is acknowledged. We gratefully appreciate generous financial support by the Bavarian State Ministry of Science, Research, and the Arts within the Collaborative Research Network "Solar Technologies go Hybrid." 
[1] B. A. Gregg, J. Phys. Chem. B 107, 4688 (2003).

[2] P. Peumans, A. Yakimov, and S. R. Forrest, J. Appl. Phys. 93, 3693 (2003).

[3] A. Bruno, L. X. Reynolds, C. Dyer-Smith, J. Nelson, and S. A. Haque, J. Phys. Chem. C 117, 19832 (2013).

[4] Y. Tamai, Y. Matsuura, H. Ohkita, H. Benten, and S. Ito, J. Phys. Chem. Lett. 5, 399 (2014).

[5] M. Orrit, J. Bernard, J. M. Turlet, and Ph. Kottis, J. Chem. Phys. 78, 2847 (1983).

[6] R. R. Lunt, J. B. Benziger, and S. R. Forrest, Adv. Mater. 22, 1233 (2010).

[7] D. Kurrle and J. Pflaum, Appl. Phys. Lett. 92, 133306 (2008).

[8] J. Wagner, M. Gruber, A. Hinderhofer, A. Wilke, B. Bröker, J. Frisch, P. Amsalem, A. Vollmer, A. Opitz, N. Koch, F. Schreiber, and W. Brütting, Adv. Funct. Mater. 20, 4295 (2010).

[9] A. K. Ghosh and T. Feng, J. Appl. Phys. 49, 5982 (1978).

[10] D. E. Markov, E. Amsterdam, P. W. M. Blom, A. B. Sieval, and J. C. Hummelen, J. Phys. Chem. A 109, 5266 (2005).

[11] A. Steindamm, M. Brendel, A. K. Topczak, and J. Pflaum, Appl. Phys. Lett. 101, 143302 (2012).

[12] N. H. Hansen, C. Wunderlich, A. K. Topczak, E. Rohwer, H. Schwoerer, and J. Pflaum, Phys. Rev. B 87, 241202(R) (2013).

[13] A. C. Dürr, N. Koch, M. Kelsch, A. Rühm, J. Ghijsen, R. L. Johnson, J.-J. Pireaux, J. Schwartz, F. Schreiber, H. Dosch, and A. Kahn, Phys. Rev. B 68, 115428 (2003).

[14] V. Stehr, R. F. Fink, B. Engels, J. Pflaum, and C. Deibel, J. Chem. Theory Comput. 10, 1242 (2014).

[15] R. R. Lunt, N. C. Giebink, A. A. Belak, J. B. Benziger, and S. R. Forrest, J. Appl. Phys. 105, 053711 (2009).
[16] B. Nickel, R. Barabash, R. Ruiz, N. Koch, A. Kahn, L. C. Feldman, R. F. Haglund, and G. Scoles, Phys. Rev. B 70, 125401 (2004).

[17] A. Kahn, N. Koch, and W. Gao, J. Polym. Sci., Part. B: Polym. Phys. 41, 2529 (2003).

[18] Y. Terao, H. Sasabe, and C. Adachi, Appl. Phys. Lett. 90, 103515 (2007).

[19] S. R. Scully and M. D. McGehee, J. Appl. Phys. 100, 034907 (2006).

[20] M. Theander, A. Yartsev, D. Zigmantas, V. Sundström, W. Mammo, M. R. Andersson, and O. Inganäs, Phys. Rev. B 61, 12957 (2000).

[21] T. Stübinger and W. Brütting, J. Appl. Phys. 90, 3632 (2001).

[22] Here we refer to an effective activation energy comprising contributions by the concentration gradient as well as by the temperature dependent diffusion constant.

[23] K. Hannewald, V. M. Stojanović, J. M. T. Schellekens, P. A. Bobbert, G. Kresse, and J. Hafner, Phys. Rev. B 69, 075211 (2004).

[24] A. H. Matsui, Pure Appl. Chem. 67, 429 (1995).

[25] F. Ortmann, F. Bechstedt, and K. Hannewald, New J. Phys. 12, 023011 (2010).

[26] R. W. Munn and W. Siebrand, J. Chem. Phys. 52, 47 (1970).

[27] A. C. Dürr, F. Schreiber, K. A. Ritley, V. Kruppa, J. Krug, H. Dosch, and B. Struth, Phys. Rev. Lett. 90, 016104 (2003).

[28] U. Heinemeyer, R. Scholz, L. Gisslén, M. I. Alonso, J. O. Ossó, M. Garriga, A. Hinderhofer, M. Kytka, S. Kowarik, A. Gerlach, and F. Schreiber, Phys. Rev. B 78, 085210 (2008).

[29] D. Zhang, A. Horneber, J. Mihaljevic, U. Heinemeyer, K. Braun, F. Schreiber, R. Scholz, and A. J. Meixner, J. Lumin. 131, 502 (2011). 